\title{
Allosteric histone methyltransferase modulators block tumour growth
}

Polycomb repressive complex 2 (PRC2), which regulates gene expression through its histone $\mathrm{H} 3$ lysine 27 trimethylation methyltransferase (H3K27me3) activity, is dysregulated in multiple human cancers and has attracted considerable interest as a therapeutic target. Two new studies, reported in Nature Chemical Biology, now present the discovery of novel allosteric PRC2 inhibitors, which inhibit tumour growth in mouse xenograft models.

PRC2 consists of three core subunits: EZH2, EED and SUZ12. Inhibitors of the catalytic subunit EZH2, which act by competing with the methyl-donating cofactor $S$-adenosylmethionine (SAM), are currently being tested in clinical trials of haematological malignancy. However, recent studies have indicated that tumour cell lines treated with SAM-competitive inhibitors can acquire secondary EZH2 mutations that render them resistant to these agents. Given the role of EED in propagating the enzymatic activity

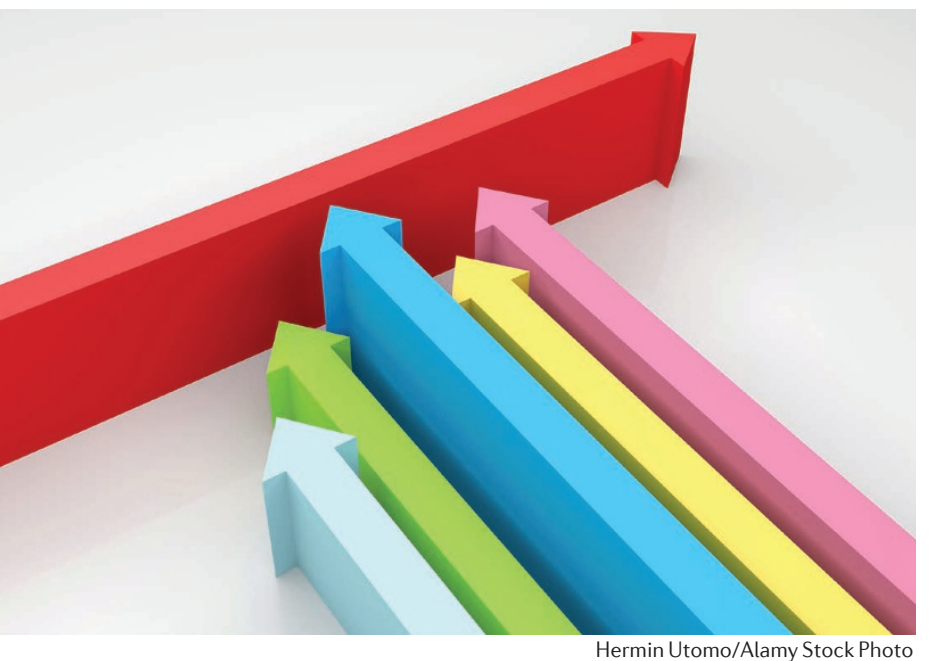

of PRC2, Qi et al. and He et al. set out to explore the potential of therapeutically targeting EED.

Qi et al. began by further characterizing and optimizing SAMnoncompetitive inhibitors identified in a previous high-throughput screen for PRC2 inhibitors, which led to the discovery of EED226. A series of in vitro assays demonstrated that EED226 is a potent and selective allosteric PRC2 inhibitor that binds to the H3K27me3 binding pocket of EED.

Determination of the highresolution crystal structure of EED in complex with EED226 and an EZH2 peptide revealed that EED226 binds to the top pocket of the $\beta$-propeller formed by seven WD40 units through which EED recognizes the H3K27me3 peptide. The binding site is composed of four aromatic residues that form an 'aromatic cage', which - upon binding of EED226 - undergoes a substantial conformational change to create a deeper pocket.

In cells, EED226 specifically inhibited H3K27 methylation and PRC2 complex activity, thereby modulating gene transcription similarly to the SAM-competitive $\mathrm{EZH} 2$ inhibitor EI1. In mice with subcutaneous lymphoma xenograft tumours, oral twice-daily dosing of EED226 for 21 days slowed tumour growth and reduced tumour volume in conjunction with decreased global $\mathrm{H} 3 \mathrm{~K} 27 \mathrm{me} 3$ levels and upregulation of target genes. When dosed for 32 days, EED226 induced complete tumour regression and was well tolerated. Notably, EED226 was able to inhibit PRC2 activity and proliferation in cells resistant to EI1.
Meanwhile, He et al. used a high-throughput thermal shift assay-based screen against the EED protein, followed by structure-activity relationship studies, which led to the identification of the pyrrolidine A-395. In vitro characterization revealed that A-395 directly binds to EED and selectively inhibits PRC2 activity.

Determination of the crystal structure of the EED WD40 domain-A-395 complex showed that, like EED226, A-395 occupied the H3K27me3 binding site, disrupting the aromatic cage, to expose a larger, more druggable binding pocket.

A-395 was confirmed to inhibit $\mathrm{H} 3 \mathrm{~K} 27$ methylation in a rhabdoid tumour cell line and showed similar antiproliferative activity profiles to EZH2 inhibitors in a panel of multiple myeloma, malignant rhabdoid tumour, lymphoma and breast cancer cell lines. Importantly, A-395 inhibited proliferation of lymphoma cell lines with acquired resistance to the EZH2 inhibitor GSK126. In vivo, in a lymphoma xenograft mouse model, subcutaneous administration of A-395 twice per week for 5 weeks inhibited tumour growth by $84 \%$ without causing toxicity.

Together, these findings indicate that targeting the $\mathrm{H} 3 \mathrm{~K} 27 \mathrm{me} 3$ pocket in EED is a promising approach for treating cancers that are dependent on PRC2 activity, particularly in patients resistant to SAM-competitive EZH2 inhibitors.

Sarah Crunkhorn

ORIGINAL ARTICLES Qi, W. et al. An allosteric PRC2 inhibitor targeting the H3K27me 3 binding pocket of EED. Nat. Chem. Biol. http://dx.doi. org/10.1038/nchembio.2304 (2017). | He, Y.et al. The EED protein-protein interaction inhibitor A-395 inactivates the PRC2 complex. Nat. Chem. Biol. http://dx.doi.org/10.1038/nchembio.2306 (2017) 